Freiburg-THEP 94/08

April 1994

\title{
Two-loop heavy Higgs corrections to the Higgs fermionic width
}

\author{
Adrian Ghinculov \\ Albert-Ludwigs-Universität Freiburg, Fakultät für Physik \\ Hermann-Herder Str.3, D-79104 Freiburg, Germany
}

\begin{abstract}
We calculate the two-loop electroweak corrections to the fermionic decay width of the Higgs boson at leading order in $m_{H}$. The sum of one-loop and two-loop radiative corrections turns out to be at $6 \%$ level over the whole range of $m_{H}$ where the perturbation theory is supposed to be a sensible approximation. We show that the perturbative approach breaks down at $m_{H} \sim 1.4 \mathrm{TeV}$, and address the question of its relevance beyond this limit.
\end{abstract}


That the selfinteraction of the electroweak symmetry breaking sector of the standard model increases with the mass of the Higgs boson, and that this eventually leads to the breakdown of the perturbation theory, belongs to the common wisdom. To establish the limits of perturbation theory is, however, a more delicate matter.

The coupling constant of the Higgs sector, $\frac{g}{4 \pi} \frac{m_{H}}{m_{W}}$, becomes of order unity for $m_{H} \sim 1.5 \mathrm{TeV}$. This is roughly where one expects the problems related to the asymptotic nature of the perturbative series to show up.

The perturbative solution for the $S$ matrix is formally unitary, but its approximations of any finite order are not. The unitarity violation effects are of higher order in the coupling constant. If the perturbation theory fails to converge satisfactorily, they become numerically large, impairing the relevance of the perturbative amplitudes. The well-known partial wave analyses of longitudinal vector boson scattering at tree level by Dicus and Mathur [1] , and B.W. Lee, C. Quigg and H. Thacker [2] show that the the unitarity bounds are exceeded for $m_{H} \sim 1 \mathrm{TeV}$. Durand, Johnson and Lopez [3, 田 extended this analysis to include the one-loop radiative corrections, and extracted a bound of $\sim 400 \mathrm{GeV}$. This much smaller value is, however, to be traced back to their criterion for unitarity violation, which is stronger than that of refs. [1, 2], rather than to genuine one-loop effects.

It is, of course, hard to draw out unambiguous conclusions about the breakdown of the perturbative series if only its first two terms are known. The first nontrivial criterion is to compare the magnitude of the one-loop and two-loop radiative corrections, and to establish where the series starts to diverge.

Here one must distinguish between radiative corrections to low energy parameters, and to processes involving the symmetry breaking scalars. The latter are expected to be larger, since the contributions which are of leading order in the Higgs mass cancel in the radiative corrections to low energy parameters [9, 8]. For instance, the two-loop radiative corrections to the $\rho$ parameter and to the vector boson selfcouplings are quadratic in $m_{H}$, up to logarithms of $m_{H}$. They become comparable to the one-loop logarithmic corrections only for Higgs bosons as heavy as 3-4 TeV [6, [].

On the other hand, performing multiloop radiative corrections to processes which involve the Higgs boson is a difficult task. Already in the case of the two-loop massive diagrams no general analytical solution exists for nonvanishing external momenta. One is therefore forced to calculate such diagrams at least partly numerically.

We calculate in this paper the leading two-loop corrections to the fermio- 
nic width of the Higgs boson, which grow quartically with $m_{H}$. We use the method described in ref. [5] to calculate the massive two-loop diagrams. The two-loop correction becomes larger than the one-loop one if $m_{H}>1.4$ $\mathrm{TeV}$, signaling the breakdown of the perturbation theory. This is compatible with the results of ref. [5], where the two-loop corrections to the shape of the Higgs resonance were calculated and shown to become large for $m_{H} \sim$ 1.2 TeV. This is also roughly the value one would expect by considering the magnitude of the coupling constant of the Higgs sector. On the other hand, this disagrees with the conclusions of ref. [10] about the breakdown of perturbation theory at $380 \mathrm{GeV}$ 円. In view of this disagreement, special emphasis is put on checks of both the analytical and numerical parts of the calculation.

Since we are interested in the leading $m_{H}$ effects, the natural choice is to work in Landau gauge. Power counting arguments show that in this gauge only the Feynman diagrams with scalar propagators survive [9, 11]. The diagrams with fermions, gauge bosons, and Fadeev-Popov fields need not be taken into account since they are not of leading order. However, we effectively evaluate the diagrams in a nearly-Landau gauge, that is, we keep a small, finite gauge parameter $\xi$ throughout the calculation, and take the limit $\xi \rightarrow 0$ in the final result. This is necessary at two-loop level in order to avoid the arbitrariness of $\int d^{n} p \frac{1}{p^{4}}$ within the dimensional regularization. One can convince oneself that this is a regular limit, in the sense that no finite contributions from the diagrams with gauge or Fadeev-Popov ghosts survive. This procedure provides also a good check of the calculation, since all $\xi$ dependence must cancel in the final results.

Within this framework, the leading corrections to the Yukawa couplings $-\frac{1}{2} i g \frac{m_{f}}{m_{W}} H f \bar{f}$ are a pure renormalization effect. They are simply given by a factor $\left(\frac{Z_{H}}{1-\frac{\delta m_{W}^{2}}{m_{W}^{2}}}\right)^{\frac{1}{2}}$, so one only needs to evaluate selfenergy diagrams.

Denoting the bare Higgs and Goldstone fields by $H_{0}, w^{ \pm}$and $z^{0}$, one writes down the Higgs sector of the standard model as:

\footnotetext{
${ }^{1}$ The two-loop radiative corrections of ref. [10] are one order of magnitude larger, which is also roughly the order of magnitude of the cancellations between different diagrams. Ref. 11] probably misses these cancellations because of a few diagrams evaluated incorrectly, two of which were already identified in ref. [5]. One also remarks that their $\partial S_{0}$, which they identify with zero, is a quantity which diverges logarithmically as the mass of the Goldstone bosons tends to zero. The proof that $\partial S_{0}=0$ given in ref. [10] is based on illegally splitting this integral into two contributions whose definition domains do not overlap.
} 


$$
\begin{aligned}
\mathcal{L}= & \frac{1}{2}\left(\partial_{\mu} H_{0}\right)\left(\partial^{\mu} H_{0}\right)+\frac{1}{2}\left(\partial_{\mu} z_{0}\right)\left(\partial^{\mu} z_{0}\right)+\left(\partial_{\mu} w_{0}^{+}\right)\left(\partial^{\mu} w_{0}^{-}\right) \\
& -g^{2} \frac{m_{H_{0}}^{2}}{m_{W_{0}}^{2}} \frac{1}{8}\left[w_{0}^{+} w_{0}^{-}+\frac{1}{2} z_{0}^{2}+\frac{1}{2} H_{0}^{2}+\frac{2 m_{W_{0}}}{g} H_{0}+\frac{4 \delta t}{g^{2} \frac{m_{H_{0}}^{2}}{m_{W_{0}}^{2}}}\right]^{2}
\end{aligned}
$$

where $\delta t$ is the tadpole counterterm needed to ensure that the vacuum expectation value of the Higgs field does not receive quantum corrections. It is related to the scalar selfenergy at zero momentum. One further splits the Lagrangian of eq. 1 into a renormalized Lagrangian and counterterms:

$$
\begin{aligned}
H_{0} & =Z_{H}^{1 / 2} H \\
z_{0} & =Z_{G}^{1 / 2} z \\
w_{0} & =Z_{G}^{1 / 2} w \\
m_{H_{0}}^{2} & =m_{H}^{2}-\delta m_{H}^{2} \\
m_{W_{0}}^{2} & =m_{W}^{2}-\delta m_{W}^{2},
\end{aligned}
$$

and fixes the counterterms by using physical, on-shell renormalization conditions with field renormalization. Note that the gauge coupling constant $g$ does not get renormalized at leading order in the coupling constant of the Higgs system $g^{2} \frac{m_{H}^{2}}{m_{W}^{2}}$. One can express $g$ in terms of the Fermi coupling constant and the mass of the charged vector boson as $g^{2}=4 \sqrt{2} m_{W}^{2} G_{F}$, with $G_{F}=1.16637 \cdot 10^{-5} \mathrm{GeV}^{-2}$, and $m_{W}=80.6 \mathrm{GeV}$.

The necessary one-loop counterterms at $\mathcal{O}(\epsilon)$ are given in ref. [5].

One can now proceed with the actual two-loop calculation. The main task is to evaluate the $\mathrm{W}$ boson selfenergy at zero momentum for extracting the $\mathrm{W}$ mass counterterm, and the on-shell derivative of the Higgs selfenergy for calculating the field renormalization constant of the Higgs boson. This involves the selfenergy topologies of. fig. 1. An algebraic computer program generates all relevant Feynman diagrams, and uses the techniques of ref. [5] to calculate them. In the case of the selfenergy of the $\mathrm{W}$ boson it is possible to obtain an analytic result because the external momentum vanishes. For the wave function renormalization of the Higgs boson, some numerical integrations have to be performed. The results are: 


$$
\begin{aligned}
\delta m_{W}^{2(2-\text { loop })=} & -\left(g^{2} \frac{m_{H}^{2}}{m_{W}^{2}}\right)^{2}\left(\frac{m_{H}^{2}}{4 \pi \mu^{2}}\right)^{\epsilon} \frac{m_{W}^{2}}{\left(16 \pi^{2}\right)^{2}}\left[\frac{3}{32} \frac{1}{\epsilon}-\frac{1}{128}+\frac{3}{32} \gamma-\right. \\
& \left.-\frac{\pi^{2}}{192}+\frac{3 \sqrt{3} \pi}{64}-\frac{3 \sqrt{3}}{16} C l\left(\frac{\pi}{3}\right)\right] \\
\delta Z_{H}^{(2-\text { loop })=} & \operatorname{Re}\left\{( g ^ { 2 } \frac { m _ { H } ^ { 2 } } { m _ { W } ^ { 2 } } ) ^ { 2 } ( \frac { m _ { H } ^ { 2 } } { 4 \pi \mu ^ { 2 } } ) ^ { \epsilon } \frac { 1 } { ( 1 6 \pi ^ { 2 } ) ^ { 2 } } \left[\frac{3}{32} \frac{1}{\epsilon}-\right.\right. \\
& -0.416(6)-i 1.000(7)]\}
\end{aligned}
$$

where $\gamma=0.577215664901532860607$ is the Euler constant, and $C l$ denotes the Clausen function, with $C l\left(\frac{\pi}{3}\right)=1.01494160640965362502$.

A number of checks were performed to make sure that these results are correct.

The cancellation of logarithms and poles in $\xi$ was checked analytically for $\delta m_{W}^{2(2-l o o p)}$, and numerically for $\delta Z_{H}^{(2-l o o p)}$. The complex integration paths for $\delta Z_{H}^{(2-l o o p)}$ were varied to make sure the integrand is indeed analytical along the integration path over the Feynman parameters (see ref. [5]). The numerical value of $\delta Z_{H}^{(2-l o o p)}$ agrees with the less precise one which can be derived from the momentum dependence of the Higgs selfenergy calculated in ref. [5]. Note that the latter result implies numerical integrations over very different functions, and is sustained by the fact that its imaginary part agrees with the one-loop corrections to the Higgs width. The imaginary part of the momentum derivative of the Higgs selfenergy, which is given in eq. 3 ,

$$
-1.000(7)\left(g^{2} \frac{m_{H}^{2}}{m_{W}^{2}}\right)^{2} \frac{1}{\left(16 \pi^{2}\right)^{2}},
$$

obtained by numerically integrating the two-loop diagrams, agrees with the analytical result which can be derived by using the Cutkosky rule:

$$
\begin{gathered}
-\left(g^{2} \frac{m_{H}^{2}}{m_{W}^{2}}\right)^{2} \frac{1}{\left(16 \pi^{2}\right)^{2}} \frac{3 \pi}{4}\left(1+\frac{\pi \sqrt{3}}{12}-\frac{5 \pi^{2}}{48}\right)= \\
=-1.002245142\left(g^{2} \frac{m_{H}^{2}}{m_{W}^{2}}\right)^{2} \frac{1}{\left(16 \pi^{2}\right)^{2}} .
\end{gathered}
$$


Further checks were performed by calculating the two-loop selfenergy of the Goldstone bosons. After performing an expansion in the external momentum, one can calculate the necessary Feynman diagrams analytically. The selfenergy of the Goldstone bosons at zero momentum agrees with the two-loop tadpole counterterm given in ref. [5], and the wave function renormalization satisfies the generalized Ward identity at leading order in $m_{H}$, $\delta m_{W}^{2}=-m_{W}^{2} \delta Z_{G}$.

We are now in the position to calculate the two-loop radiative corrections to the Higgs fermionic width:

$$
\begin{aligned}
\frac{Z_{H}}{Z_{G}}= & \frac{1+\delta Z_{H}^{(1-\text { loop })}+\delta Z_{H}^{(2-\text { loop })}+\ldots}{1+\delta Z_{G}^{(1-\text { loop })}+\delta Z_{G}^{(2-l o o p)}+\ldots} \\
= & 1+\delta Z_{H}^{(1-\text { loop })}-\delta Z_{G}^{(1-\text { loop })}+\delta Z_{G}^{(1-\text { loop })}\left(\delta Z_{G}^{(1-l o o p)}-\right. \\
& \left.-\delta Z_{H}^{(1-\text { loop })}\right)+\delta Z_{H}^{(2-\text { loop })}-\delta Z_{G}^{(2-\text { loop })}+\ldots
\end{aligned}
$$

where

$$
\begin{aligned}
\delta Z_{H}^{(1-\text { loop })} & =g^{2} \frac{m_{H}^{2}}{m_{W}^{2}}\left(\frac{m_{H}^{2}}{4 \pi \mu^{2}}\right)^{\epsilon / 2} \frac{1}{16 \pi^{2}}\left[\frac{3}{2}-\frac{\pi \sqrt{3}}{4}+\mathcal{O}(\epsilon)\right] \\
\delta Z_{G}^{(1-\text { loop })} & =g^{2} \frac{m_{H}^{2}}{m_{W}^{2}}\left(\frac{m_{H}^{2}}{4 \pi \mu^{2}}\right)^{\epsilon / 2} \frac{1}{16 \pi^{2}}\left[-\frac{1}{8}+\mathcal{O}(\epsilon)\right] .
\end{aligned}
$$

Numerically, one obtains:

$$
\frac{Z_{H}}{Z_{G}}=1+.264650 \frac{g^{2}}{16 \pi^{2}} \frac{m_{H}^{2}}{m_{W}^{2}}-.303(8)\left(\frac{g^{2}}{16 \pi^{2}} \frac{m_{H}^{2}}{m_{W}^{2}}\right)^{2}+\ldots .
$$

The results are shown in fig. 2 .

Including the two-loop results reduces the magnitude of the radiative corrections, wich remain under $6 \%$ for the whole range of validity of the perturbative approach, up to $\sim 1.4 \mathrm{TeV}$, where the two-loop correction exactely compensates the one-loop one.

Finally, some comments on the possible relevance of perturbation theory beyond this limit are in order. In an attempt to sum the asymptotic series of eq. 6 , fig. 3 shows the behaviour of the first nontrivial term of its Shanks transformation. Since the perturbative series is a power series, this 
reduces actually to the Padé approximant [1/1]. Certainly, the convergence radius of the perturbative expansion is zero, and a diagonal sequence of Padé approximants might fail to sum it. To sum the perturbative series would require knowledge of its high order behaviour. On the much simpler case of the anharmonic oscillator one can show that finding the right nonlinear sequence transformation to sum an asymptotic series depends crucially on the knowledge of an estimate of the series' remainder 13. If such information is not available, the only thing one can do is to compare the results of different summation recipes [12], but, of course, knowledge of only the oneloop and two-loop corrections does not leave much room for experimenting with nonlinear sequence transformations.

\section{Acknowledgement}

The author wishes to thank prof. Jochum van der Bij for useful discussions.

\section{References}

[1] D.A. Dicus and V.S. Mathur, Phys. Rev. D7 (1973) 3111.

[2] B.W. Lee, C. Quigg and H. Thacker, Phys. Rev. D16 (1977) 1519.

[3] L. Durand, J.M. Johnson and J.L. Lopez, Phys. Rev. Lett. 64 (1990) 1215.

[4] L. Durand, J.M. Johnson and J.L. Lopez, Phys. Rev. D45 (1992) 3112.

[5] A. Ghinculov and J.J. van der Bij, Freiburg-THEP 94/05.

[6] J.J. van der Bij and M. Veltman, Nucl. Phys. B231 (1984) 205.

[7] J.J. van der Bij, Nucl. Phys. B255 (1985) 648.

[8] M. Veltman, Acta Phys. Pol. B8 (1977) 475.

[9] M. Einhorn and J. Wudka, Phys. Rev. D39 (1989) 2758.

[10] P.N. Maher, L. Durand and K. Riesselmann, Phys. Rev. D48 (1993) 1061 ; Phys. Rev. D48 (1993) 1084 ; L. Durand, B.A. Kniehl and K. Riesselmann, DESY 93-131.

[11] W.J. Marciano and S.S.D. Willenbrock, Phys. Rev. D37 (1988) 2509. 
[12] E.J. Weniger, Comp. Phys. Rep. 10 (1989) 189.

[13] E.J. Weniger, J. Čížek and F. Vinette, J. Math. Phys. 34 (1993) 571. 


\section{Figure captions}

Fig.1 The topologies of the two-loop selfenergy diagrams.

Fig. 2 The radiative corrections $\frac{Z_{H}}{Z_{G}}$ to the fermionic Higgs width in the one-loop (solid line) and two-loop (dashed line) approximations as a function of the mass of the Higgs boson.

Fig. 3 The behaviour of the Padé approximant [1/1] (dotted line) in the strong coupling zone, compared to the one-loop (solid line) and two-loop (dashed line) approximations. 
This figure "fig1-1.png" is available in "png" format from: http://arxiv.org/ps/hep-ph/9405394v1 
This figure "fig1-2.png" is available in "png" format from: http://arxiv.org/ps/hep-ph/9405394v1 
This figure "fig1-3.png" is available in "png" format from: http://arxiv.org/ps/hep-ph/9405394v1 